\title{
VcFT-induced mobile florigenic signals in transgenic and transgrafted blueberries
}

\author{
Guo-qing Song ${ }^{1}$, Aaron Walworth', Tianyi Lin', Qiuxia Chen', Xiumei Han², L. Irina Zaharia² and Gan-yuan Zhong ${ }^{3}$
}

\begin{abstract}
FLOWERING LOCUS T (FT) can promote early flowering in annual species, but such role has not been well demonstrated in woody species. We produced self and reciprocal grafts involving non-transgenic blueberry (NT) and transgenic blueberry (T) carrying a 35S-driven blueberry FT (VCFT-OX). We demonstrated that the transgenic VCFT-OX rootstock promoted flowering of non-transgenic blueberry scions in the NT (scion):T (rootstock) grafts. We further analyzed RNASeq profiles and six groups of phytohormones in both NT:T and NT:NT plants. We observed content changes of several hormone metabolites, in a descending order, in the transgenic NT:T, non-transgenic NT:T, and non-transgenic NT:NT leaves. By comparing differential expression transcripts (DETs) of these tissues in relative to their control, we found that the non-transgenic NT:T leaves had many DETs shared with the transgenic NT:T leaves, but very few with the transgenic NT:T roots. Interestingly, a number of these shared DETs belong to hormone pathway genes, concurring with the content changes of hormone metabolites in both transgenic and non-transgenic leaves of the NT:T plants. These results suggest that phytohormones induced by VCFT-OX in the transgenic leaves might serve as part of the signals that resulted in early flowering in both transgenic plants and the non-transgenic NT:T scions.
\end{abstract}

\section{Introduction}

Florigen was originally defined to be graft-transmissible hormones or hormone-like molecules involved in longdistance regulation of flowering ${ }^{1-3}$. Initially, florigen was hypothesized to be a specific ratio of known hormones and metabolites despite a lack of convincing molecular evidence $^{1-3}$. With the discovery of FLOWERING LOCUS $T(F T)$ in Arabidopsis ${ }^{4,5}$, and functional analysis of $F T$ or its orthologues in many plant species ${ }^{6-8}$, and subsequent demonstration of $F T$ as a promoter of flowering with short-distance mobility from leaves to adjacent meristems $^{9-11}, F T$ emerged as a top candidate for florigen.

However, accepting "FT-as-florigen" is still controversial. The main contentious issue is the mechanism by which FT signals are transmitted from source leaf to

\footnotetext{
Correspondence: Guo-qing Song (songg@msu.edu) or

Gan-yuan Zhong (GanYuan.Zhong@ARS.USDA.GOV)

${ }^{1}$ Plant Biotechnology Resource and Outreach Center, Department of Horticulture, Michigan State University, East Lansing, MI 48824, USA

${ }^{2}$ Aquatic and Crop Resource Development, National Research Council of Canada, Saskatoon SK S7N OW9, Canada

Full list of author information is available at the end of the article.
}

recipient meristems to promote flowering. For herbaceous plants, many reports have demonstrated that FT proteins, instead of $F T$ RNAs, acted as the mobile florigenic signals mainly by short-distance transport (e.g., from leaves to their adjacent buds) ${ }^{9,12-19}$. Two other reports, however, suggested that both FT proteins and FT RNAs could be transmitted $^{20,21}$. For woody plants, the evidence for FTbased flowering promotion and FT proteins or FT RNAs as florigenic signals is not consistent. For example, in woody shrubs, while overexpression of the Jatropha FT in transgenic Jatropha rootstock promoted flowering in recipient scions ${ }^{22}$, such a phenomenon was not observed in recipient scions grafted on transgenic cassava (Manihot esculenta Crantz) containing a constitutively expressed Arabidopsis $F T^{23}$. In both reports, potential transport of Jatropha FT proteins or FT RNAs as florigenic signals were not analyzed ${ }^{22,23}$. Various attempts to promote flowering in recipient scions by expressing $F T$ or $F T$ orthologues from poplar (Populus trichocarpa PpFT) in transgenic rootstocks of poplar ${ }^{24}$, apple ${ }^{25}$, and plum $^{26}$ have been unsuccessful. Among these studies, PpFT 
mRNAs from transgenic rootstocks were only detected in non-transgenic scions of micro-grafted apple plants ${ }^{25}$. Very disappointedly, no further studies on long-distance transport of FT proteins or $F T$ mRNAs in transgrafted woody plants have been reported. Equally disappointed is that no satisfactory explanations are available for why overexpression of transgenic $F T$ in rootstocks could not promote flowering of non-transgenic scions in woody species ${ }^{9}$.

While florigen was initially believed to be hormones or hormone-like molecules, surprisingly, little connection has been made between them. It is well known that phytohormones [e.g., abscisic acid (ABA), auxin, cytokinin, ethylene, and gibberellins (GAs)] play important roles in regulating plant flowering and stature formation ${ }^{27}$. For example, mutations resulting in reduced GA biosynthesis or increased GA degradation often produce dwarf plants with delayed flowering ${ }^{28-32}$. Other phytohormone genes [e.g., $\operatorname{auxin}^{33,34}$, cytokinin $^{3,35,36}$, ethylene ${ }^{37}$, brassinosteroid $^{38,39}$, jasmonic acid $(\mathrm{JA})^{40}$, nitric oxide ${ }^{41}$, peptide hormone $^{42}$, and salicylic acid $(\mathrm{SA})^{43,44}$ ] also affect plant flowering and size. Because of small molecular sizes and diverse physiological properties, phytohormones are known to be an important class of biochemical signals in plant development and growth

To understand FT-based flowering promotion in woody species, we previously generated transgenic blueberry with overexpressed $V c F T$ ( $V c F T$-OX) and showed $V c F T$ induced flowering in transgenic blueberry ${ }^{45}$. In this study, our objective was to investigate whether VcFT-OX plants can be used as rootstocks to induce flowering in nontransgenic scions and, if yes, what would likely be the florigenic signals moving from rootstocks to scions to promote flowering. Through grafting, we demonstrated the ability of the transgenic $V c F T$ blueberry, when it was uses as a rootstock, to induce flowering of non-transgenic blueberry scions. We further revealed that transgenic $V c F T$ was highly expressed in transgenic leaves, but surprisingly suppressed in the transgenic roots. Moreover, we identified several hormone genes, among others, differentially expressed and the expression levels of some of these genes corelated with the content changes of corresponding hormones between the transgenic rootstock and non-transgenic scion. We concluded that phytohormones were likely involved in $F T$-mediated flowering and could be a part of the florigenic signals moving from leaves to adjacent buds or apical meristems for inducing flowering in blueberry.

\section{Results}

VCFT-OX promoted flowering in transgrafted scions

We previously demonstrated that overexpression of $V c F T$-induced precocious flowering and dwarfing in all transgenic blueberry 'Aurora' plants ${ }^{46}$. We subsequently analyzed the gene expression patterns of a representative transgenic event (hereafter noted as VcFT-OX-Aurora) and revealed numerous differentially expressed genes (DEGs) associated with flowering and phytohormone pathways ${ }^{45,47}$. Due to the well-defined phenotypic changes and the availability of the micropropagated plants, in this study, 3-year-old plants of this representative transgenic VcFT-OX-Aurora ( $\mathrm{T}$ ) and non-transgenic 'Aurora' (NT) were self [i.e., T (scion):T (rootstock) and NT:NT] and reciprocally (i.e., NT:T and T:NT) grafted. Ungrafted 6-year-old NT plants with flower buds, as expected, did not flower without fulfilling chilling requirement (Fig. 1a), while ungrafted, nonchilled $\mathrm{T}$ plants showed flowering, although some abnormal flowers with wrinkled petals were observed (Fig. 1b, c). The fully chilled $\mathrm{T}$ plants, however, had well-formed flowers and flowered normally (Fig. 1e, f), compared with the 6-year-old NT plants (Fig. 1d). The fully chilled 3-year-old ungrafted and NT:NT plants did not flower due to the lack of flower buds.

Grafting is not a common practice for blueberry bushes, but it has potential to increase abiotic stress tolerance for cultivated blueberry cultivars ${ }^{48}$. In this study, the survival rate of grafted scions on $\mathrm{T}$ rootstocks ranged from 16.7 to $60.0 \% 2$ weeks after grafting. In contrast, all T:NT grafting failed. The survived transgenic scions on T:T plants were similar to the ungrafted $\mathrm{T}$ plants, producing no new shoots due to the lack of leaf buds. In contrast, the survived non-transgenic scions on both NT:T and NT:NT plants formed new shoots after four and half months (Fig. 2). Under non-chilling conditions, none of the NT shoots on $\mathrm{T}$ rootstocks produced flowers. However, after receiving full chilling ( 1600 chilling hours) for 2 months and transferring to a warm greenhouse, flowering was observed from buds on the NT shoots of all the six NT:T plants within 2 weeks. In contrast, no flowers were observed on the NT scions (grafted or non-grafted) on any NT:NT plants or on ungrafted 'Aurora' plants (Fig. 2, Fig. S1). Apparently, the NT:T plants were able to promote flowering of non-transgenic scions when exposed to chilling. These results suggested that the VcFT-OX in the transgenic rootstock of the NT:T plants initiated florigenic signals from either transgenic leaves or transgenic roots that went through a long-distance transportation for hastening flower bud formation and flowering in the nontransgenic scions.

\section{VCFT-OX altered the contents of some major phytohormones}

Florigen was initially believed to be hormones or hormone-like molecules ${ }^{2}$. In order to determine potential roles of phytohormones in promoting flowering, 41 metabolites of six phytohormone groups [ABA (7 metabolites), auxin (6), cytokinin (10), GA (14), JA (3), and SA (1)] were quantified in both transgenic and non- 

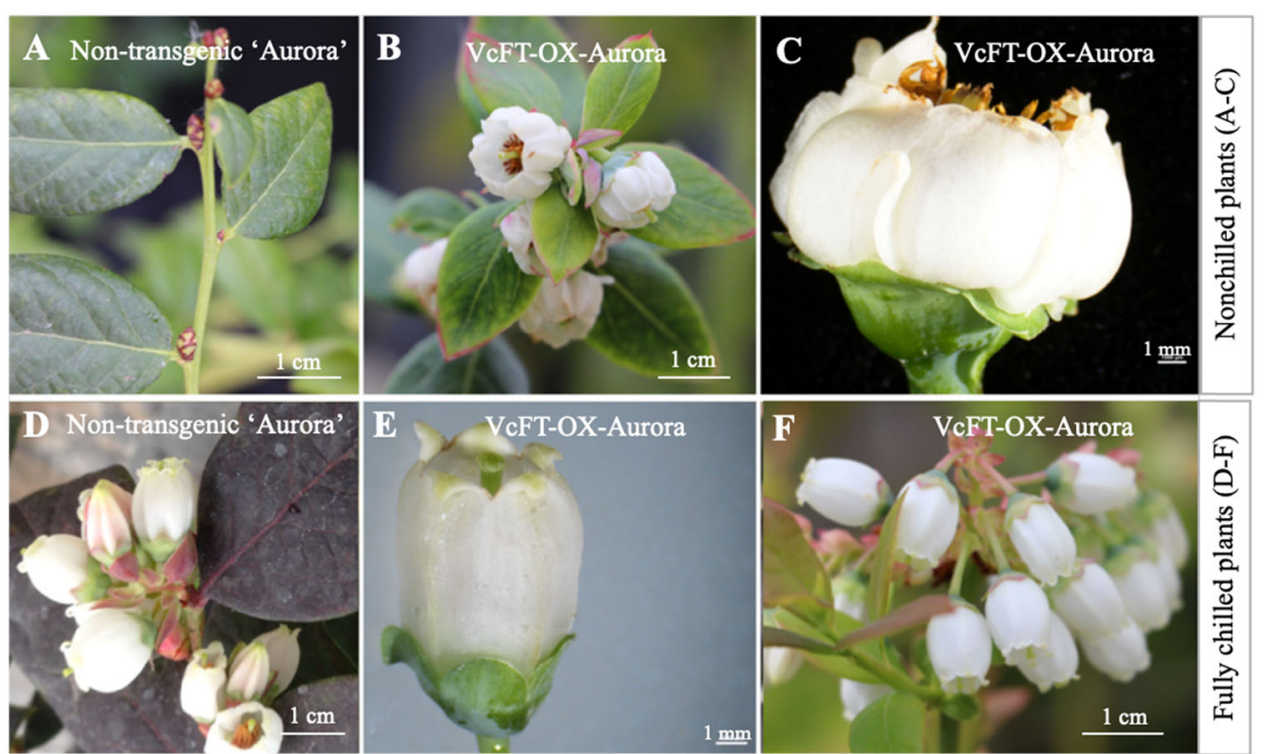

Fig. 1 Flowers of 6-year-old, non-transgenic 'Aurora' and 3-year-old VcFT-OX-Aurora plants. a Nonchilled 'Aurora' flower buds. b, c Flowers of nonchilled VcFT-OX-Aurora. d Flowers of fully chilled non-transgenic 'Aurora'. e, f Flowers of fully chilled VcFT-OX-Aurora. Note: few flower buds appeared in the 3-year-old VCFT-OX 'Aurora' plants
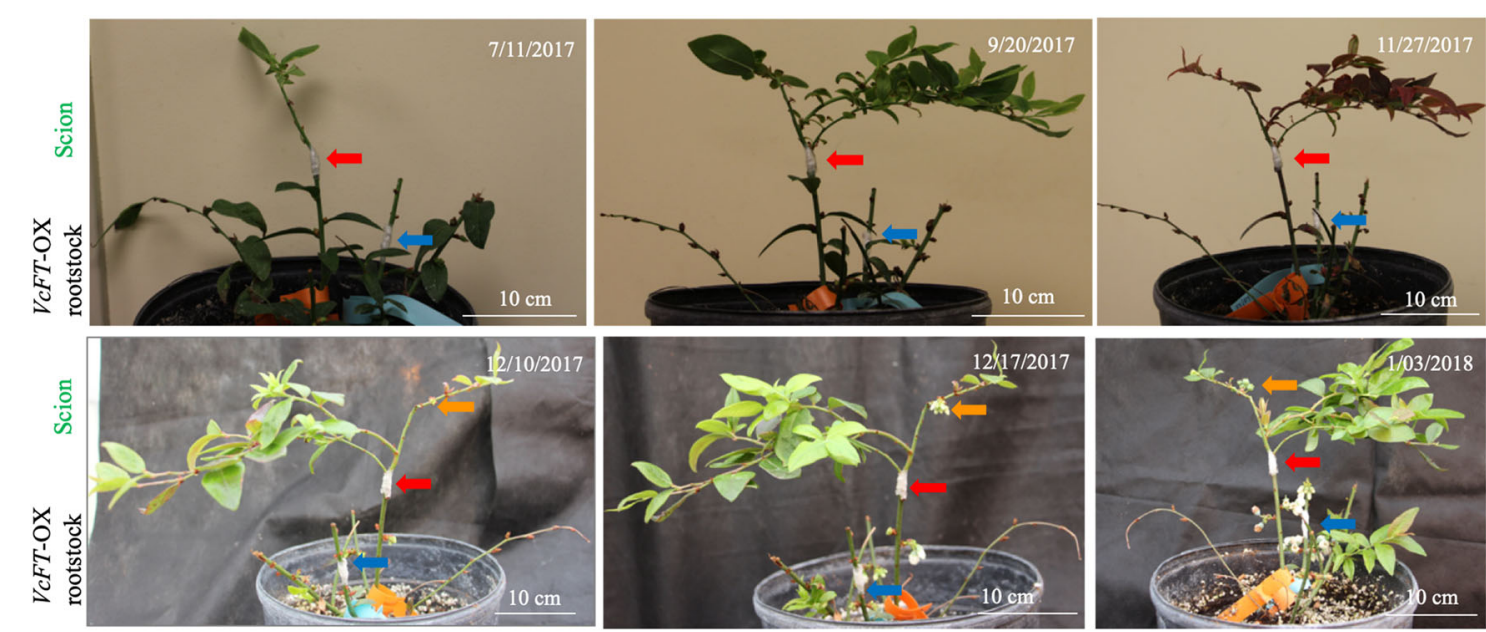

Fig. 2 A VcFT-OX-Aurora plant with the graft combinations of T:T and NT:T. The shown T:T and NT:T grafting was made on May 6, 2017; after receiving chilling treatment from September 20 to November 27, 2017, flower buds on the non-transgenic NT scion were observed to break on December 10, 2017. Red, blue, and orange arrows show the grafting unions of NT:T, T:T and the flowers or fruits on the NT scion, respectively. No flowers were observed in non-transgenic 'Aurora' NT or NT:NT plants during the whole observation period from November 27, 2017 to May 23, 2018 (Fig. S1)

transgenic tissues of the NT:T plants as well as in the control plants NT:NT.

We analyzed seven ABA and ABA metabolites and they were all detected in both transgenic and non-transgenic leaves of NT:T plants (note that transgenic leaves were from the transgenic rootstocks $\mathrm{T}$ and non-transgenic leaves from the grafted non-transgenic scions NT) as well as in the non-transgenic NT:NT leaves. The total accumulation level of $\mathrm{ABA}$ and its metabolites in the transgenic leaves was higher than those in the nontransgenic leaves from the NT:T plants, but the trend varied among individual metabolites (Fig. 3a), with dihydrophaseic acid (DPA) and phaseic acid (PA) showing lower accumulation in the transgenic leaves. The reduction of DPA in the transgenic leaves was statistically significant $(p=0.01)$ (Fig. 3, Table 1). Overall accumulation 


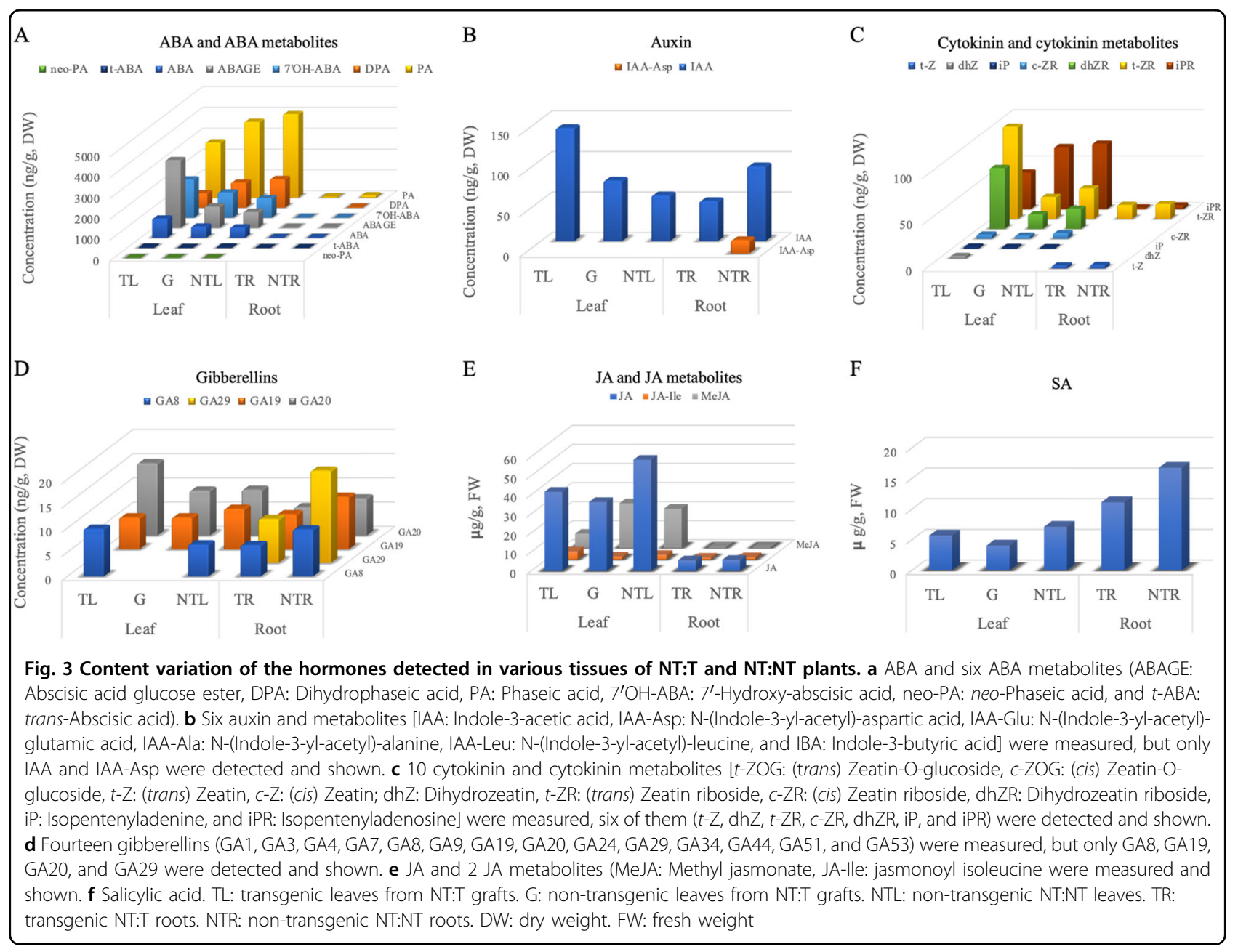

of the seven ABA metabolites was much lower in the roots than in the leaves. All seven metabolites were present in NT:NT roots, and six of them, except for DPA, were detected in the transgenic NT:T roots (Fig. 3, Table $1)$. ABA showed a significant decrease $(p=0.02)$ in the transgenic NT:T roots and the total level of ABA and its metabolites in the transgenic roots was about half of that observed in the non-transgenic roots (Fig. 3, Table 1). All seven ABA and ABA metabolites were also detected in the transgenic NT:T leaves, although none of them had significant accumulation changes compared with the nontransgenic leaves of both NT:T and NT:NT plants $(p=$ 0.05) (Table 1). However, the accumulation levels of the seven metabolites were mostly between transgenic and non-transgenic leaves of the $\mathrm{NT} / \mathrm{T}$, with three being increased and two decreased (Fig. 3a).

We assayed six auxins and only indole-3-acetic acid (IAA) and $\mathrm{N}$-(indole-3-yl-acetyl)-aspartic acid (IAA-Asp) were detected (Fig. 3b, Table 1). Biologically active IAA was found in all of the leaf and root samples while IAAAsp was detected only in the non-transgenic roots. In the
NT:T plants, IAA accumulation was the highest in transgenic leaves, followed by non-transgenic leaves and transgenic roots. In the NT:NT plants, roots had higher IAA content than leaves. When NT:T plants were compared with NT:NT plants, non-transgenic NT:T leaves had higher IAA content than the NT:NT leaves $(p=0.06)$. However, IAA content in the transgenic roots showed no significant difference $(p=0.28)$ from the non-transgenic root samples, despite the apparently higher IAA content in the non-transgenic roots $(92.3 \mathrm{ng} / \mathrm{g} \mathrm{DW}$ in the nontransgenic roots versus $49.0 \mathrm{ng} / \mathrm{g} \mathrm{DW}$ in the transgenic roots) (Table 1). This was likely due to the small sample size in the analysis. The IAA accumulation in the nontransgenic NT:T leaves was intermediate between that of the transgenic NT:T leaves and non-transgenic NT:NT leaves.

Cytokinins were the top candidate for florigen before the discovery of $F T^{3,4}$. Seven cytokinins were measured in this study. Six of them were detected in leaves and three in roots (Fig. 3c, Table 1). Of the three cytokinins detected in roots, isopentenyladenosine content showed a significant 
Table 1 Statistical analysis of the content variation of the hormones detected in various tissues of NT:T and NT: NT plants

\begin{tabular}{|c|c|c|c|c|c|c|}
\hline \multirow[b]{2}{*}{ Hormone group } & \multirow[b]{2}{*}{$\begin{array}{l}\text { Hormones and } \\
\text { metabolites }\end{array}$} & \multicolumn{5}{|l|}{ ng/g dry weight } \\
\hline & & $\begin{array}{l}\text { Transgenic NT: } \\
\text { T leaves }\end{array}$ & $\begin{array}{l}\text { Non-transgenic NT: } \\
\text { T leaves }\end{array}$ & $\begin{array}{l}\text { Non-transgenic NT: } \\
\text { NT leaves }\end{array}$ & $\begin{array}{l}\text { Transgenic NT: } \\
\text { T roots }\end{array}$ & $\begin{array}{l}\text { Non-transgenic NT: } \\
\text { NT roots }\end{array}$ \\
\hline \multirow[t]{7}{*}{ ABA } & $A B A$ & $927.3 \pm 451.8 a^{*}$ & $544.7 \pm 174.5 \mathrm{a}$ & $499.7 \pm 88.2 \mathrm{a}$ & $18.7 \pm 1.2 c$ & $23.7 \pm 2.1 b$ \\
\hline & DPA & $697.3 \pm 91.6 b$ & $1204.0 \pm 110.6 \mathrm{a}$ & $1371.0 \pm 262.1 \mathrm{a}$ & nd & $7.7 \pm 13.3 \mathrm{C}$ \\
\hline & ABAGE & $3252.0 \pm 1862.9 \mathrm{a}$ & $1023.0 \pm 110.0 \mathrm{a}$ & $763.3 \pm 188.9 \mathrm{a}$ & $25.3 \pm 4.7 b$ & $37.7 \pm 8.3 b$ \\
\hline & PA & $2645.7 \pm 618.2 \mathrm{a}$ & $3635.7 \pm 535.9 \mathrm{a}$ & $4010.0 \pm 1030.1 \mathrm{a}$ & $43.7 \pm 21.1 b$ & $110.3 b$ \\
\hline & $7^{\prime} \mathrm{OH}-\mathrm{ABA}$ & $1833.0 \pm 1561.2 \mathrm{a}$ & $1213.0 \pm 418.8 \mathrm{a}$ & $934.3 \pm 96.1 \mathrm{a}$ & $8.9 \pm 4.5 b$ & $19.3 \pm 9.3 b$ \\
\hline & neo-PA & $8.0 \pm 4.4 \mathrm{a}$ & $6.7 \pm 4.9 \mathrm{a}$ & $4.7 \pm 0.8 \mathrm{a}$ & nd & nd \\
\hline & $t-A B A$ & $50.3 \pm 22.0 \mathrm{a}$ & $25.3 \pm 18.3 \mathrm{a}$ & $54.3 \pm 20.6 \mathrm{a}$ & $5.3 \pm 0.6 b$ & $4.7 \pm 0.6 b$ \\
\hline \multirow[t]{7}{*}{ Cytokinin } & $t-Z R$ & $99.7 \pm 23.2 \mathrm{a}$ & $24.0 \pm 8.9 b$ & $33.0 \pm 18.1 b$ & $15.3 \pm 2.5 c$ & $16.0 \pm 6.6 c$ \\
\hline & $c-Z R$ & $4.7 \pm 0.6 b$ & $3.3 \pm 1.5 b$ & $6.3 \pm 1.1 \mathrm{a}$ & nd & nd \\
\hline & dhZR & $65.7 \pm 15.3 \mathrm{a}$ & $15.7 \pm 5.9 b$ & $21.3 \pm 8.1 b$ & nd & nd \\
\hline & iP & $1.7 \pm 0.6 \mathrm{a}$ & $1.7 \pm 0.6 \mathrm{a}$ & $1.3 \pm 0.6 \mathrm{a}$ & nd & nd \\
\hline & iPR & $39.7 \pm 2.1 \mathrm{a}$ & $67.0 \pm 15.6 \mathrm{a}$ & $70.7 \pm 33.3$ a & $1.7 \pm 0.6 b$ & $4.0 \pm 1.0 \mathrm{C}$ \\
\hline & $t-Z$ & nd & nd & nd & $3.3 \pm 1.2 \mathrm{a}$ & $4.0 \pm 1.0 \mathrm{a}$ \\
\hline & $d h Z$ & $1.0 \pm 1.7 \mathrm{a}$ & nd & nd & nd & nd \\
\hline \multirow[t]{2}{*}{ Auxin } & IAA & $139.0 \pm 41.6 \mathrm{a}$ & $74.7 \pm 28.4 \mathrm{ab}$ & $56.3 \pm 35.9 \mathrm{ab}$ & $49.0 \pm 41.2 b$ & $92.3 \pm 44.1 \mathrm{ab}$ \\
\hline & IAA-Asp & nd & nd & nd & nd & $16.3 \pm 10.1 b$ \\
\hline \multirow[t]{5}{*}{ GA } & GA20 & $15.0 \pm 6.9 \mathrm{a}$ & $9.3 \pm 4.2 \mathrm{a}$ & $9.7 \pm 7.4 \mathrm{a}$ & $6.0 \pm 1.0 \mathrm{a}$ & $6.3 \pm 4.0 \mathrm{a}$ \\
\hline & GA29 & nd & nd & nd & $3.0 \pm 5.2 \mathrm{a}$ & $9.7 \pm 8.2 \mathrm{a}$ \\
\hline & GA8 & $6.7 \pm 7.0 \mathrm{a}$ & $1.3 \pm 2.3 \mathrm{a}$ & $3.9 \pm 3.6 \mathrm{a}$ & $5.7 \pm 2.1 \mathrm{a}$ & $6.1 \pm 3.4 \mathrm{a}$ \\
\hline & GA19 & $5.6 \pm 1.8 \mathrm{a}, \mathrm{b}$ & $3.5 \pm 3.3 b$ & $6.7 \pm 2.5 \mathrm{a}, \mathrm{b}$ & $7.3 \pm 2.1 \mathrm{a}, \mathrm{b}$ & $10.7 \pm 1.2 \mathrm{a}$ \\
\hline & & $\mu \mathrm{g} / \mathrm{g}$ fresh weight & & & & \\
\hline SA & SA & $5.7 \pm 1.6 \mathrm{a}$ & $4.0 \pm 1.8 \mathrm{a}$ & $7.1 \pm 3.7 \mathrm{a}$ & $11.1 \pm 5.2 \mathrm{a}, \mathrm{b}$ & $16.7 \pm 4.5 \mathrm{~b}, \mathrm{c}$ \\
\hline \multirow[t]{3}{*}{$J A$} & $J A$ & $41.5 \pm 15.1 \mathrm{a}, \mathrm{b}$ & $36.2 \pm 16.3 \mathrm{a}, \mathrm{b}$ & $58.3 \pm 18.2 \mathrm{a}$ & $5.6 \pm 2.5 \mathrm{c}$ & $5.8 \pm 1.9 \mathrm{C}$ \\
\hline & JA-lle & $4.2 \pm 2.1 \mathrm{a}$ & $1.5 \pm 0.5 \mathrm{a}$ & $2.4 \pm 1.1 \mathrm{a}$ & $0.9 \pm 1.3 \mathrm{a}$ & $1.2 \pm 0.2 \mathrm{a}$ \\
\hline & MeJA & $7.4 \pm 3.6 b$ & $23.4 \pm 2.9 \mathrm{a}$ & $20.5 \pm 4.5 \mathrm{a}$ & $0.4 \pm 0.2 c$ & $0.3 \pm 0.1 c$ \\
\hline
\end{tabular}

Nd non-detectable

*Numbers (means of three biological replicates \pm standard deviation) in each row with different letters are significantly different at $p=0.05$

decrease in the transgenic roots compared with the nontransgenic roots. Of the six cytokinins detected in leaves, all were detected in the transgenic leaves and five in the non-transgenic leaves of both NT:T and NT:NT plants. The content of dihydrozeatin riboside (dhZR) and (trans) zeatin riboside $(\mathrm{t}-\mathrm{ZR})$ was significantly higher in the transgenic NT:T leaves than in the non-transgenic leaves of either NT:T and NT:NT plants (Table 1).

Gibberellins play significant roles in regulating plant flowering ${ }^{49,50}$. The content of 14 GAs were measured, but only 3 and 4 were detected in the leaf and root samples, respectively (Fig. 3d, Table 1). No significant changes of
GAs were found either between transgenic leaves and non-transgenic leaves or between transgenic roots and non-transgenic roots. Compared with non-transgenic NT: NT leaves, transgenic leaves appeared to have less GA19, but more GA8 and GA20, and transgenic roots had less GA19, GA20, and GA29 than non-transgenic roots. In the non-transgenic NT/T leaves, GA19 and GA20, but not GA8, were detected (Table 1).

The hormones of JA and two JA metabolites (Fig. 3e) and SA (salicylic acid, Fig. 3f) were detected in both leaf and root samples (Table 1). Relative to the non-transgenic NT:NT leaves, MeJA showed a significant decrease in 
transgenic leaves while SA was significantly decreased in transgenic roots. Notably, none of the four compounds measured showed a significant difference between transgenic and non-transgenic leaves of the NT:T plants.

\section{VcFT-OX induced genome-wide differential gene expression}

To examine VcFT-OX induced transcriptomic changes, RNA-Seq profiles of non-transgenic NT:NT leaves, transgenic and non-transgenic NT:T leaves were compared (Fig. S2, Table S1). The comparison between nontransgenic NT:NT leaves and transgenic NT:T leaves resulted in 2421 DE transcripts (DETs) (948 DEGs: hereafter the DEGs refer to the differentially expressed unique genes defined by the Trinotate annotation) (FDR $<0.05$ ), of which $V c F T$ was about 5000-fold (FPKM = 107.70) greater in transgenic NT:T leaves compared with non-transgenic NT:NT leaves $(\mathrm{FPKM}=0.02)$ and 962 DETs (420 DEGs) were upregulated in the transgenic leaves (Table S1). In comparison, 1165 DETs (723 DEGs) were found between non-transgenic NT:NT leaves and non-transgenic NT:T leaves, of which only 318 DETs (162 DEGs) showed increased expression in the non-transgenic NT:T leaves (Table S1). A total of 299 DETs (150 DEGs) were overlapped between the two comparisons, and the expression changes ( $\log _{2} \mathrm{FC}$ values) of these 299 DETs exhibited a strong positive correlation $(y=0.8078 \times$ $-0.2905, R=0.82, p$-value $<4.4 \mathrm{e}-4)$ between the two groups (Fig. 4a, Table S2).

In a previous study, we observed in the blueberry cultivar 'Legacy' that VcFT in nonchilled flower buds had higher expression $(\mathrm{FPKM}=34.9)$ than young leaves (FPKM: 0.2) and flowers (FPKM: 0.9) ${ }^{47}$. Similarly, in this study, non-transgenic NT:NT plants had low VcFT expressions in leaves (FPKM: 0.02) and roots (FPKM: 0.08). The VcFT expression in the transgenic leaves and roots of NT:T plants were 107.70 and 0.12 FPKM, respectively. The accumulation of $V c F T$ RNA messages in the transgenic roots was surprisingly low, but in line with the general observation that $F T$ expression is much suppressed in roots ${ }^{51}$. Transcriptome comparison between the transgenic and non-transgenic roots resulted in 18,275 DETs of 5516 genes (Table S1). Since VcFT expression were very low in both transgenic and nontransgenic roots, such many DETs observed between their comparisons (Table S1) was very intriguing. A common set of 517 DETs of 274 genes were identified in the comparison between the 723 DEGs in non-transgenic NT: $\mathrm{T}$ leaves and 18,275 DETs in transgenic NT:T roots (Fig. S2). The expression changes ( $\log _{2} \mathrm{FC}$ values) of these 517 DETs showed a very weak positive linear correlation $(y=$ $-0.1685 \times+0.7667, R=0.15, p$-value $<2.2 \mathrm{e}-16)$ between two groups of the DETs (Fig. 4b), suggesting that the changes of the 517 DETs in non-transgenic NT:T leaves

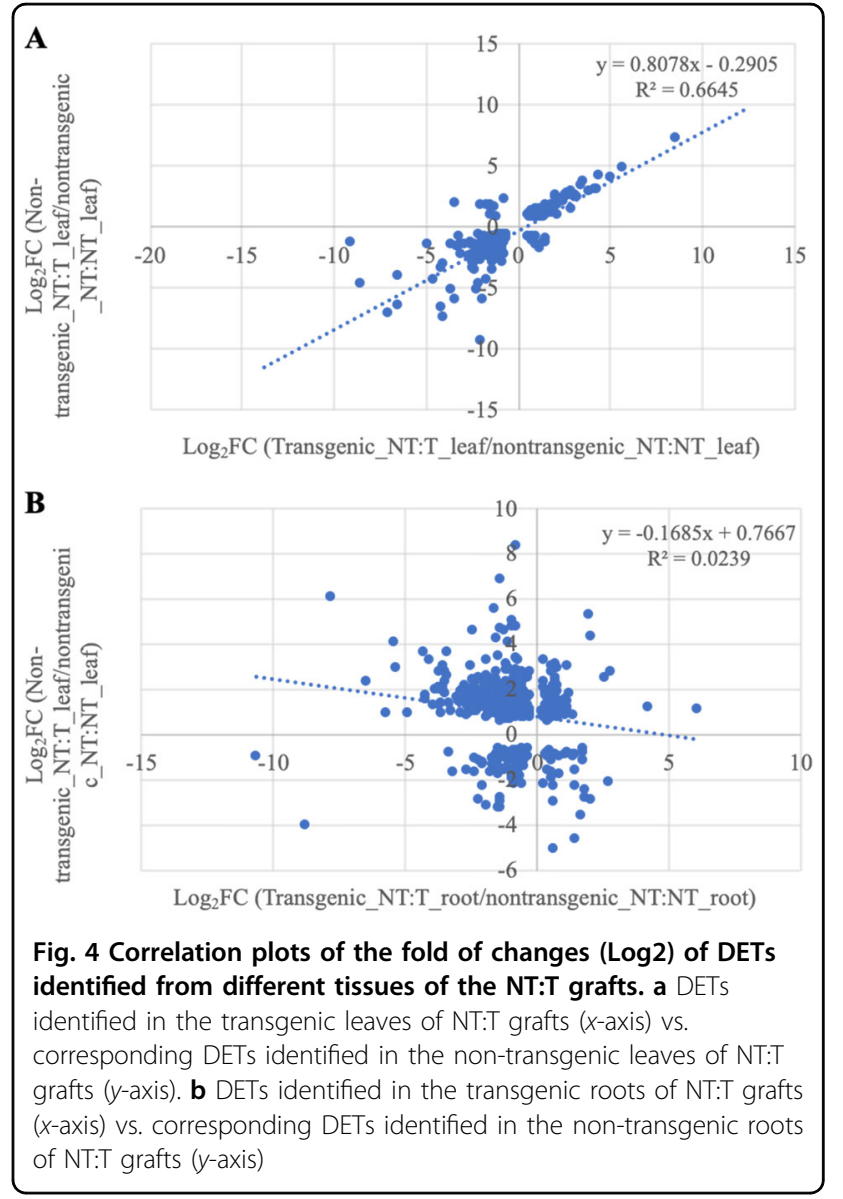

were not much related to the DETs from transgenic roots. A gene network analysis of the 299 DETs shared in the comparisons of non-transgenic NT:NT leaves versus transgenic and non-transgenic NT:T leaves, respectively, revealed 91 gene ontology (GO) terms. Among them, 18 were related to flower development and 7 related to hormones (Fig. S3, Tables S2, S3), indicating that hormone pathway genes were significantly involved in the $V c F T$-triggered flowering processes.

In the differential expression analysis, we applied a very restrictive FDR value to declare significances for various comparisons. We found that many transcripts which were not expressed in one of the paired tissues in the comparisons often did not pass the cut-off threshold. However, these transcripts may represent some genes which were uniquely suppressed or enhanced in the tissue because of the introduction of the VcFT. We examined these transcripts and found 760 which were expressed in the non-transgenic NT:NT leaves (all three biological replicates with FPKM >0.1) but suppressed in the transgenic leaves (all three replicates with FPKM $=0)($ Table S4). In contrast, 216 were not detected in the nontransgenic NT:NT leaves but expressed in the transgenic leaves. Similarly, 193 transcripts were expressed in the 
Table 2 Differentially expressed transcripts of flowering pathway genes detected in the non-transgenic leaves of NT:T vs. NT:NT plants

\begin{tabular}{llllllll}
\hline Query_id & Subject_id & E-value & Log 2 FC & $\boldsymbol{p}$-value & FDR & Annotation by Trinotate & Gene_name \\
\hline AT5G13480.2 & C88585_g1_i1 & $3.00 E-20$ & -1.07 & $2.68 E-07$ & 0.00013 & COB23_ARATH & FY \\
AT5G24470.1 & C92704_g6_i2 & $2.00 E-116$ & -2.09 & $3.56 E-10$ & 0.00000 & PRR95_ORYSJ & APRR5, PRR5 \\
AT5G24470.1 & C92704_g6_i1 & $1.00 E-116$ & -2.23 & $1.05 E-08$ & 0.00001 & APRR5_ARATH & APRR5, PRR5 \\
AT4G22950.1 & C94107_g4_i1 & $7.00 E-33$ & -2.35 & $1.38 E-07$ & 0.00007 & SOC1_ARATH & AGL19, GL19 \\
AT4G34000.1 & C89508_g1_i2 & $3.00 E-50$ & -1.68 & $6.37 E-08$ & 0.00004 & Al5L5_ARATH & ABF3, DPBF5 \\
AT2G45660.1 & C94107_g3_i3 & $1.00 E-23$ & -6.54 & $6.13 E-05$ & 0.01222 & - & AGL20, SOC1 \\
AT2G23080.1 & C98469_g3_i1 & $7.00 E-22$ & -0.85 & $4.17 E-04$ & 0.05358 & Y1461_ARATH & AT2G23080.1 \\
AT1G13260.1 & C83982_g1_i2 & $6.00 E-130$ & -1.24 & $2.64 E-06$ & 0.00095 & RAV1_ARATH & RAV1, EDF4 \\
AT2G45660.1 & C88293_g4_i5 & $3.00 E-34$ & -2.84 & $1.02 E-06$ & 0.00041 & - & AGL20, SOC1 \\
AT4G22950.1 & C94107_g4_i2 & $1.00 E-20$ & -4.64 & $8.67 E-06$ & 0.00255 & SOC1_ARATH & AGL19, GL19 \\
\hline
\end{tabular}

$\log _{2} F C \log _{2}$ fold change (NT:T/NT:NT), CPM count per million reads, FDR false discovery rate

non-transgenic NT:T leaves, but suppressed in the nontransgenic NT:NT leaves while expression behavior of 14 other transcripts were reversed in the two tissues. Interestingly, 187 (177 suppressed and 10 enhanced) of the 207 transcripts of the non-transgenic NT:T leaves (193 suppressed and 14 enhanced) were shared with the 876 uniquely suppressed or enhanced transcripts in the transgenic leaves (760 suppressed and 216 enhanced). In other words, about $90 \%$ of the uniquely suppressed or enhanced transcripts in the non-transgenic NT:T leaves were found in the transgenic leaves, which accounted for about $21 \%$ of the total uniquely suppressed or enhanced transcripts in the transgenic leaves. On the other hand, only 25 transcripts $(<3 \%)$ were shared between the transgenic leaves (876) and transgenic roots of the NT:T plants (1480). To our surprise, there were no other overlaps of such uniquely suppressed or enhanced transcripts between non-transgenic leaves and transgenic roots of the NT:T plants, except for two (suppressed) which were shared by all three tissues. These results strongly suggested that DETs in the NT:NT leaves were much more related to those in the transgenic leaves and had little connection with those in the transgenic roots. Since many of these shared transcripts could not be annotated with the current known databases, their functions and significance in promoting flowering are unknown.

\section{Differential expression of flowering, hormones, and sugar pathway genes}

By using flowering pathway genes of Arabidopsis as inquiries ${ }^{47}$, we found 41 DETs in the differential expression analysis of transgenic leaves vs. non-transgenic NT: NT leaves, 10 DETs in the non-transgenic NT:T leaves vs. non-transgenic NT:NT leaves, and 262 DETs in the transgenic roots vs. non-transgenic roots (Table S5). Among the 10 DETs in the NT:T leaves, 5 DETs (4 DEGs) were shared with the transgenic leaves (Table 2). One of them is SOC1 which is a well-known target of $F T$ in flowering ${ }^{52}$. Interestingly, all four genes showed much reduced expression in both transgenic and non-transgenic leaves of the NT:T plants, compared with the nontransgenic NT:NT leaves. On the other hand, there was only one DEG, AT5G13480.2, shared between the nontransgenic leaves and transgenic roots in the NT:T plants. AT5G13480.2 is a known regulator in flowering ${ }^{53}$. There were eight flowering-related DETs shared between the transgenic leaves and transgenic roots. Similarly, we found 20 DETs of sugar pathway genes in the comparison of transgenic NT:T leaves vs. non-transgenic NT:NT leaves, 13 DETs in the non-transgenic NT:T leaves vs. nontransgenic NT:NT leaves, and 164 DETs in the transgenic roots vs. non-transgenic roots (Table S6). Most of these DETs were downregulated in the transgenic tissues. Surprisingly, there was no overlapped sugar DETs between the transgenic and non-transgenic leaves in the NT:T plants, while 6 and 10 were shared between the nontransgenic leaves and transgenic roots and between the transgenic leaves and transgenic roots in the NT:T plants, respectively. In contrast to the flowering and sugar genes, by using the reference of the 245 biosynthetic pathway genes representing nine groups of phytohormones (i.e., ABA, auxin, brassinosteroid, cytokinin, gibberellin, ethylene, JA, SA, and strigolactones) of $A$. thaliana (http:// hormones.psc.riken.jp), we found 98, 71, and 463 DETs of hormone pathway genes in the transgenic leaves, nontransgenic leaves, and transgenic roots of the NT:T plants, respectively (Table 3 ). There were 23 DETs of hormone genes shared between non-transgenic and transgenic leaves, 34 between non-transgenic leaves and transgenic 
Table 3 Differentially expressed transcripts of phytohormone genes detected in the comparisons of transgenic leaves from NT:T vs. non-transgenic leaves from NT:NT, non-transgenic leaves from NT:T vs. non-transgenic leaves from NT:NT, and transgenic roots from NT:T vs. non-transgenic roots form NT:NT

\begin{tabular}{|c|c|c|c|c|c|c|c|}
\hline Query id & Subject id & $\begin{array}{l}\log _{2} \text { (NT:T } \\
\text { transgenic leaves/ } \\
\text { NT:NT leaves) }\end{array}$ & $\begin{array}{l}\log _{2}(\mathrm{NT}: \mathrm{T} \\
\text { non- } \\
\text { transgenic } \\
\text { leaves/NT: } \\
\text { NT leaves) }\end{array}$ & $\begin{array}{l}\log _{2}(\mathrm{NT}: \mathrm{T} \\
\text { root/ NT: } \\
\text { NT root) }\end{array}$ & $\begin{array}{l}\text { Annotation by } \\
\text { Trinotate }\end{array}$ & Hormone & Gene_name \\
\hline AT2G29090.1 & c80869_g4_i1 & $\# \mathrm{~N} / \mathrm{A}$ & -1.79 & -0.68 & ABAH1_ARATH & ABA & CYP707A2 \\
\hline AT2G29090.1 & c84314_g2_i1 & $\# \mathrm{~N} / \mathrm{A}$ & -1.63 & $\# N / A$ & ABAH1_ARATH & ABA & CYP707A2 \\
\hline AT2G29090.1 & c88313_g2_i1 & -1.37 & -2.58 & $\# N / A$ & ABAH1_ARATH & $\mathrm{ABA}$ & CYP707A2 \\
\hline AT1G52400.1 & C80508_g1_i1 & $\# N / A$ & 1.46 & $\# N / A$ & BGL34_ORYSJ & $\mathrm{ABA}$ & BGL1, ATBG1, BGLU18 \\
\hline AT1G52340.1 & C84480_g1_i1 & -1.83 & -1.14 & -1.86 & C7A29_PANGI & $\mathrm{ABA}$ & SRE1, ISI4, ABA2 \\
\hline AT3G19270.1 & c80869_g4_i2 & $\# \mathrm{~N} / \mathrm{A}$ & -1.95 & -1.13 & C7A52_PANGI & ABA & CYP707A4 \\
\hline AT3G19270.1 & c83078_g3_i3 & $\# N / A$ & -2.19 & $\# N / A$ & C7A52_PANGI & $A B A$ & CYP707A4 \\
\hline AT5G45340.1 & c88211_g1_i2 & $\# \mathrm{~N} / \mathrm{A}$ & -1.61 & -1.85 & C7A52_PANGI & $\mathrm{ABA}$ & CYP707A3 \\
\hline AT1G30100.1 & C99178_g3_i1 & \#N/A & -1.11 & -0.77 & NCED1_PHAVU & $\mathrm{ABA}$ & NCED5, ATNCED5 \\
\hline AT1G52340.1 & C84480_g1_i2 & $\# \mathrm{~N} / \mathrm{A}$ & -1.71 & -2.84 & SILD_FORIN & $\mathrm{ABA}$ & SRE1, ISI4, ABA2 \\
\hline AT4G31500.1 & c93443_g1_i2 & $\# N / A$ & -1.54 & -0.66 & C71E1_SORBI & Auxin & CYP735A1 \\
\hline AT4G31500.1 & c97022_g2_i1 & \#N/A & -1.72 & $\# N / A$ & C71E1_SORBI & Auxin & CYP701A3, ATKO1, GA3 \\
\hline AT2G30770.1 & C91695_g3_i1 & $\# N / A$ & -1.65 & -1.62 & C78A7_ARATH & Auxin & CYP701A3, ATKO1, GA3 \\
\hline AT2G30770.1 & c91695_g3_i3 & $\# \mathrm{~N} / \mathrm{A}$ & -1.74 & $\# \mathrm{~N} / \mathrm{A}$ & C78A7_ARATH & Auxin & CYP701A3, ATKO1, GA3 \\
\hline AT2G30770.1 & C99171_g4_i6 & -0.87 & -1.09 & $\# N / A$ & C78A7_ARATH & Auxin & CYP701A3, ATKO1, GA3 \\
\hline AT4G32540.1 & c83588_g1_i1 & 1.26 & 1.27 & $\# \mathrm{~N} / \mathrm{A}$ & YUC_ARATH & Auxin & YUC, YUC1 \\
\hline AT1G17060.1 & C93255_g4_i1 & 1.15 & 0.81 & 1.8 & C7A29_PANGI & $\mathrm{BR}$ & CYP735A1 \\
\hline AT2G26710.1 & C93255_g4_i5 & $\# \mathrm{~N} / \mathrm{A}$ & -1.56 & $\# \mathrm{~N} / \mathrm{A}$ & C7A29_PANGI & $B R$ & CYP735A1 \\
\hline AT5G38450.1 & c100283_g2_i2 & 0.67 & -0.86 & -0.94 & C14A1_ARATH & Cytokinin & CYP735A1 \\
\hline AT2G36750.1 & c88918_g1_i2 & $\# N / A$ & -2.04 & -2.09 & SCGT_TOBAC & Cytokinin & UGT73C1 \\
\hline AT2G36800.1 & C96045_g2_i1 & $\# N / A$ & -1.74 & $\# N / A$ & U91A1_ARATH & Cytokinin & UGT73C5, DOGT1 \\
\hline AT1G22400.1 & c86370_g7_i2 & 1.71 & 1.58 & $\# N / A$ & UGT2_GARJA & Cytokinin & UGT85A1, ATUGT85A1 \\
\hline AT1G22400.1 & C98889_g4_i2 & \#N/A & -1.55 & $\# N / A$ & UGT2_GARJA & Cytokinin & UGT85A1, ATUGT85A1 \\
\hline AT1G22400.1 & C98889_g4_i4 & $\# \mathrm{~N} / \mathrm{A}$ & -3.08 & -4.88 & UGT2_GARJA & Cytokinin & UGT85A1, ATUGT85A1 \\
\hline AT1G15550.1 & C89534_g1_i1 & $\# \mathrm{~N} / \mathrm{A}$ & -4.28 & -1.52 & ACCO1_ORYSI & GA & GA4, ATGA3OX1, GA3OX1 \\
\hline AT1G80340.1 & C93875_g1_i4 & $\# \mathrm{~N} / \mathrm{A}$ & -1.06 & -1.5 & DIOX3_PAPSO & GA & ACO1, ATACO1 \\
\hline AT4G25420.1 & c54669_g1_i1 & \#N/A & -5.34 & $\# N / A$ & FL3H_PETCR & GA & ATGA200X1, GA5, GA200X1 \\
\hline AT4G25420.1 & c81615_g1_i2 & $\# N / A$ & -6.7 & $\# N / A$ & FL3H_PETCR & GA & ACO1, ATACO1 \\
\hline AT4G25420.1 & c94454_g1_i1 & 1.77 & 0.79 & -0.65 & FL3H_PETCR & GA & ACO1, ATACO1 \\
\hline AT4G02780.1 & c97468_g5_i1 & $\# \mathrm{~N} / \mathrm{A}$ & -0.89 & $\# N / A$ & NES2_FRAAN & GA & ATCPS1, CPS, CPS1, GA1 \\
\hline AT4G21200.1 & C89719_g1_i1 & -1.61 & -2.04 & -1.75 & SILD_FORIN & GA & ACO1, ATACO1 \\
\hline AT4G21200.1 & c89719_g1_i2 & $\# \mathrm{~N} / \mathrm{A}$ & -3.02 & -1.21 & SILD_FORIN & GA & ATACO2, ACO2 \\
\hline AT1G20510.1 & c72977_g1_i1 & $\# \mathrm{~N} / \mathrm{A}$ & -2.01 & -1.42 & 4CL2_SOYBN & $J A$ & OPCL1 \\
\hline AT1G20510.1 & c98993_g2_i2 & $\# N / A$ & -0.96 & $\# N / A$ & 4CL2_SOYBN & $J A$ & OPCL1 \\
\hline AT2G27690.1 & C79501_g3_i1 & $\# N / A$ & -2.16 & $\# N / A$ & C86A2_ARATH & $J A$ & CYP94C1 \\
\hline AT2G27690.1 & c8721_g1_i1 & $\# \mathrm{~N} / \mathrm{A}$ & -2.06 & -1.8 & C86A2_ARATH & $J A$ & CYP94C1 \\
\hline AT2G06050.2 & c98433_g1_i3 & $\# \mathrm{~N} / \mathrm{A}$ & -0.14 & 0.43 & OPR11_ORYSJ & $J A$ & OPR3, DDE1, ATOPR3 \\
\hline AT1G22400.1 & c90644_g3_i6 & 2.8 & 2.44 & $\# \mathrm{~N} / \mathrm{A}$ & KGLT_PETHY & SA & UGT85A1, ATUGT85A1 \\
\hline AT2G23620.1 & C83239_g2_i1 & $\# \mathrm{~N} / \mathrm{A}$ & -1.53 & $\# \mathrm{~N} / \mathrm{A}$ & SABP2_TOBAC & SA & MES1, ATMES1 \\
\hline
\end{tabular}

$\log _{2} F C \log _{2}$ fold change, $\# N / A$ no differential expression, CPM count per million reads, FDR false discovery rate

roots, and 51 between transgenic leaves and transgenic roots in the NT:T plants. Interestingly, four DETs were shared among all three tissues, involving one $\mathrm{ABA}$, one cytokinin, and two GA genes (Table 3).

Among the three groups of flowering, hormone and sugar genes, many DETs (23) of hormone genes overlapped between non-transgenic and transgenic leaves of the NT:T plants suggesting that hormone genes might be involved in promoting flowering in the non-transgenic NT:T scions. Among these 23 shared DETs, there were four DETs representing two DE cytokinin genes similar to the CYP735A1 and UGT85A1 genes in Arabidopsis. CYP735A1 catalyzes biosynthesis of tZ-type cytokinins and increase in CYP735A1 expression can enhance shoot growth in A. thaliana ${ }^{54}$. UGT85A1 is involved in transzeatin homeostasis and trans-zeatin responses and 
decreased expression of $U G T 85 A 1$ can lead to a low level of the trans-zeatin O-glucosides ${ }^{55}$. In Arabidopsis, cytokinins, auxin, and sugar are all involved in flower development and fertility ${ }^{56}$. In sweet cherry, a recent study has shown that the upregulated cytokinins played an inductive role in bud dormancy release ${ }^{57}$. Whether or not the two downregulated cytokinin genes were directly responsible for the reduced accumulation of $c-Z R$ in the nontransgenic NT:T leaves remains to be resolved. Similarly, the functional roles of c-ZR in regulating plant flowering time control remains to be uncovered ${ }^{58}$.

Among the 23 DETs shared between transgenic and nontransgenic NT:T leaves, 4 were involved in the gibberellin biosynthesis pathway and they were annotated to be similar to two Arabidopsis genes, GA2ox8 and GA2ox1. GA2ox8 hydroxylates $\mathrm{C}_{20}$-GA precursors, and a repressed expression of GA20x8 promotes flowering ${ }^{59-61}$. Interestingly, GA2ox8 was downregulated in both transgenic and nontransgenic leaves of the NT:T plants in this study, which is consistent with the expected expression change of the gene for promoting flowering in the scion. Also, in agreement is the GA20ox 1 which was upregulated in both transgenic and non-transgenic NT:T leaves. In A thaliana, suppression of GA20ox in long days had little effect on flowering time, whereas in short days flowering was delayed ${ }^{62}$. Suppressed expression of GA200x1 would expect to lead to reduction of the synthesis of GA1 and then GA $8^{63}$. Indeed, we observed the reduction of GA8 content in the non-transgenic NT:T leaves and the promotion of flower bud formation in the non-transgenic NT:T shoots.

ABA is known to suppress flowering ${ }^{27}$. Two DETs representing two ABA genes, CYP707A2 and $A B A 2$, were identified in both transgenic and non-transgenic NT:T leaves. Both were downregulated, which would expect to result in increasing ABA content ${ }^{64}$. Indeed, we observed in non-transgenic NT:T leaves, as described earlier, that the content of ABA, ABA-GE, 7'-Hydroxy-abscisic acid ( $7^{\prime}$ $\mathrm{OH}-\mathrm{ABA})$, and neo-phaseic acid (neo-PA) were increased, but the content of DPA, PA, and trans-abscisic acid ( $\mathrm{t}$ ABA) were decreased, although not statistically significant.

Six DETs representing two auxin genes, CYP71A13 and $Y U C$, were among the 23 DETs shared by both transgenic and non-transgenic NT:T leaves. CYP71A13 was downregulated while YUC was upregulated. CYP71A13 catalyzes the conversion of indole-3-acetaldoxime to indole-3acetonitrile (IAN) and its upregulation is expected to enhance the production of LAN and IAA ${ }^{65}$. On the other hand, downregulated YUC1 represses the conversion of indole-3-pyruvic acid to $\mathrm{IAA}^{66,67}$. The expression behavior of YUC1 was consistent with the increased IAA content in the non-transgenic NT:T leaves.

A CYP72C1 gene in the brassinosteroids biosynthesis pathway was upregulated in both transgenic and nontransgenic NT:T leaves. This gene, along with others, can inactivate brassinosteroids ${ }^{68,69}$ and consequently lead to delayed flowering in $A$. thalian $a^{70}$. Unfortunately, the content of brassinosteroids was not assayed and therefore we could not make a connection between the expression of this gene and the changes of the brassinosteriods content in this study.

In the SA biosynthesis pathway, UGT74F1 is involved in the conversion of SA or UDP-glucose to either SAglucoside or SA glucose ester ${ }^{71}$ while MES1 (MES1_ARATH) catalyzes the conversion of methyl salicylate (MeSA) to salicylic acid ${ }^{72}$. We observed increased expression of UGT74F1 in both transgenic and non-transgenic leaves of the NT:T plants and reduced expression of MES1 in the transgenic leaves, which agrees with the fact that the SA content in the non-transgenic NT:T leaves was much lower than that in the NT:NT leaves. Surprisingly, early flowering in the transgenic VcFT-OX plants was associated with decreased SA content, which was inconsistent with the previous reports in A. thaliana where increase of SA promotes flowering ${ }^{43}$.

Six DETs of three JA biosynthesis pathway genes (i.e., CY94C1, OPR3, and OPCL1) were detected and showed reduction of expression in the non-transgenic NT:T leaves. However, none of these DEGs showed differential expressions in the transgenic leaves. CYP94C1 is known to convert 12-hydroxy-JA-Ile (12OH-JA-Ile) to the carboxy-derivative $12 \mathrm{COOH}-\mathrm{JA}-\mathrm{Ile}^{73,74}$ and therefore downregulation of CYP94C1 could increase JA and JA-Ile synthesis by reducing their use as a source of precursors ${ }^{73}$. Indeed, we observed a decrease of the content of JA and JA-Ile in the non-transgenic NT:T leaves. OPR3 and OPCL1, on the other hand, catalyze the formation of the JA precursors cyclopentane-1-octanoic acid (OPC-8:0) and OPDA-CoA and OPC-8:0-CoA, respectively ${ }^{75,76}$. Their downregulation would lead to an increase in methyl jasmonate synthesis, which was the case in the nontransgenic NT:T leaves.

There are 48 additional DETs of hormone genes which were found in the non-transgenic NT:T leaves, but not in the transgenic leaves (Table S4). Likewise, there were 75 DETs of hormone genes which were found in the transgenic leaves, but not in the non-transgenic NT:T leaves (Table S4). Not being able to detect the presence of these DETs in both tissues could be due to some biological reasons, but experimental errors and limited statistical power could also be a plausible explanation.

\section{Discussion}

\section{Transgenic leaves are necessary for promoting early flowering}

We previously demonstrated that constitutive expression of a $V c F T$ in tobacco resulted in early flowering and plant dwarfing ${ }^{46}$. However, when the VcFT-expressing tobacco plants, with their leaves removed, were used as 
rootstocks, they did not significantly change flowering time of non-transgenic tobacco scions, clearly demonstrating that transgenic roots were not able to promote early flowering of the non-transgenic scions ${ }^{77}$. This observation is consistent with the results of NT:T grafting plants in cassava ${ }^{23}$, poplar ${ }^{24}$, apple ${ }^{25}$, and plum ${ }^{26}$ in which transgenic rootstocks with overexpression of $F T$ or $F T$ orthologues did not promote flowering in the recipient scions. In contrast, two NT:T woody shrubs, including blueberry in this study and Jatropha described in a previous report ${ }^{22}$, have shown that transgenic rootstocks with overexpression of $F T$ or $F T$ orthologues were able to promote flowering of NT scions. In both cases, transgenic leaves and branches were maintained when the plants were used as graft rootstocks, suggesting that these transgenic leaves are necessary for inducing flowering of the non-transgenic scions. This observation, consistent with the long-hold belief that leaves are the primary sources of florigen signals in inducing flowering ${ }^{2}$, provides a likely explanation for why previous NT:T grafting using transgenic $F T$ rootstocks for promoting flowering of nontransgenic scions in woody species has often not been successful. Therefore, maintaining some leaves in a transgenic FT rootstock is necessary for successful induction of flowering in non-transgenic scions in a woody species.

\section{Suppressed VCFT-OX in roots}

FT expression was scarcely detected in Arabidopsis roots due to its low level of accumulation ${ }^{78,79}$. In a previous study, we found that transgenic $V c F T$ driven by a $35 \mathrm{~S}$ promoter in transgenic blueberries was expressed at a much higher level in flower buds than in developing leaves and flowers ${ }^{47}$. In this study, we further observed that $V c F T$-OX transcripts in the transgenic young leaves $(F P K M=107.7)$ were about 1000-fold higher than in the transgenic roots (FPKM: 0.12) and 5000-fold higher than that in the leaves of non-transgenic blueberries. The lack of a significant increase of $V c F T$ expression in the transgenic roots is very intriguing (transgenic roots FPKM: 0.12 vs. non-transgenic roots FPKM: 0.08 ), as a $35 \mathrm{~S}$ promoter is well-known to be able to drive expression of a variety of genes in roots ${ }^{80}$. The possible explanations for this observation may include, but not be limited to, rootspecific suppression of $V c F T$ expression and/or rapid degradation of the transcribed VcFT-OX messages. Indeed, there are several potential layers of regulation existing in plants for regulation of $F T$ expression, including those at transcriptional and posttranscriptional levels ${ }^{81}$.

\section{Florigenic signals in blueberries}

So far, there is no solid evidence to support the hypothesis that FT/FT-like protein or mRNA is the florigen that undergoes long-distance transport through grafting unions from rootstocks to scions to promote flowering in woody plants. In this study, we observed 5000-fold higher expression of $V c F T$ in the transgenic leaves than in the non-transgenic leaves. However, such a high level of $V c F T$ expression in the transgenic leaves did not have an apparent impact on the accumulation level of $V c F T$ in the non-transgenic leaves of NT:T plants. This observation confirmed the previous studies that FT RNAs do not likely function as the florigen which is transmitted to promote flowering. We did not measure $V c F T$ proteins in this study and therefore do not have direct evidence that $V c F T$ proteins were transmitted from the transgenic leaves to the non-transgenic leaves and buds of the NT:T plants for inducing flowering of the non-transgenic scions. However, the fact that many genes were differentially expressed in the non-transgenic leaves of NT:T plants and, most importantly, that non-transgenic scions on the NT:T blueberry were induced to flower clearly suggest that some florigenic signals, potentially including $V c F T$ proteins, were transmitted.

The molecular process for how FT/FT-like proteins transmits from leaves to flower organs and regulate flowering pathway genes has been documented in annual species such as Arabidopsis ${ }^{12}$. A critical step in the process is that the transmitted FT proteins partner with FD proteins, interact with $S O C 1, A P 1, L F Y$ and others, and then trigger a cascade of changes of the expression of flowering pathway genes promoting flowering. In this study, we observed five such flowering DETs (based on the Arabidopsis flowering pathway genes) differentially expressed in both transgenic and non-transgenic leaves of the NT:T plants, including SOC1 which is the direct target of FT. In addition to the flowering pathway genes, expression of 23 hormone pathway genes were also significantly affected by transgenic VcFT-OX in both transgenic leaves and non-transgenic NT:T leaves. Accompanying these hormone-related differentially expressed genes, the content of some corresponding phytohormones in the transgenic and non-transgenic NT: $\mathrm{T}$ leaves, compared with the non-transgenic NT:NT leaves, were also changed. This raises an interesting question about whether these hormones are part of the $F T$-based florigenic signals in promoting flowering.

Phytohormones affect plant flowering ${ }^{1,3,33,82}$ and have long been proposed as potential florigenic signals in promoting flowering ${ }^{3}$. However, such hormones have not been explicitly identified. Of the 41 phytohormone metabolites measured, 25 were detected in leaf tissues and some of them showed apparent content differences between transgenic NT:T leaves and non-transgenic NT: NT leaves. Interestingly, such differences for these metabolites were also observed between non-transgenic NT:T leaves and non-transgenic NT:NT leaves, although 
only GA8 and cis-zeatin riboside (c-ZR) showed statistically significant reduction in the non-transgenic NT:T leaves. However, the general trend is apparent that the non-transgenic NT:T leaves had intermediate levels of accumulation of these hormone metabolites between transgenic NT:T leaves and non-transgenic NT:NT leaves.

While we cannot make direct connections between the changed hormones content in the non-transgenic NT:T leaves and early flowering of buds on the non-transgenic NT:T scions, this study clearly demonstrated the involvement of hormones in the flowering promotion process. Whether the changed hormones content in the nontransgenic NT:T leaves resulted from direct migration of hormones from the transgenic NT:T leaves or from the activities of the hormone genes in the non-transgenic NT: $\mathrm{T}$ leaves regulated by potential transgenic $V c F T$ proteins transported from the transgenic NT:T leaves cannot be determined in this study. Given that a generally low amount of FT protein is transported from leaves to buds in promoting flowering ${ }^{12}$ and there was an apparent gradient of hormone accumulation between the transgenic and non-transgenic NT:T leaves, we suspect that certain hormones from the transgenic leaves likely migrated into the non-transgenic NT:T leaves and then to buds to promote flowering of the non-transgenic NT:T scions in this study. This hypothesis may also explain why so many differentially expressed genes were identified in the differential expression analysis of the transgenic roots versus the non-transgenic roots, but no apparent increase of the FT RNAs was found in the transgenic roots. One likely explanation is that the hormones induced by transgenic $V c F T$ in the transgenic leaves transmitted to roots and induced a cascade of changes of gene expression in the transgenic roots.

In this study, we have also observed some sugar synthesis pathway genes differentially expressed in transgenic leaves, transgenic roots, and non-transgenic NT:T leaves, indicating that sugar was likely involved in the $V c F T$-OX induced early flowering as well. Sugar is well known to affect plant flowering ${ }^{83-87}$. For example, in horticultural trees and bushes, sucrose modulates hormonal signaling in controlling bud outgrowth in Rosa hybrida ${ }^{84}$ and carbohydrates have been reported to serve as either a floral stimulus or an energy source in promoting flower bud formation ${ }^{88}$. The role of carbohydrates in regulating plant flowering is considered to be through flowering pathway genes (e.g., $F T$ and SOC1) and phytohormones (e.g., GAs and JAs) ${ }^{50,87}$.

In conclusion, this study demonstrated that overexpression of $V c F T$ in a transgenic blueberry promoted its early flowering and such transgenic blueberry, when used as a rootstock, promoted flowering of non-transgenic scions grafted to it. Previous failures for inducing flowering of non-transgenic scions by transgenic $F T$ rootstocks in woody species are likely resulted from no accumulation of enough $F T$ and/or $F T$-based flowering induction signals from the rootstocks whose leaves are usually not maintained. While $V c F T$ proteins from the transgenic, overexpressed $V c F T$ could still be the florigen in explaining early flowering of the grafted, nontransgenic scions in the present study, movement of the $V c F T$ proteins from transgenic leaves to roots for explaining the extensive alteration of gene expression in the transgenic roots, in which transgenic VcFT expression was not enhanced, seems not likely on the basis of the known functions of the proteins. Unless proved otherwise, we suspect that the $V c F T$-induced changes of phytohormones in the transgenic leaves might be responsible, at least in part, for the observed molecular and phenotypic changes in both non-transgenic scions and transgenic roots of the NT:T plants.

\section{Materials and methods Transgrafting}

Northern highbush blueberry cultivar 'Aurora' needs over 1000 chill units through winter dormancy for bud break. Non-transgenic 'Aurora' and transgenic 'Aurora' containing an overexpressed $V c F T$ were used in blueberry transgrafting experiments. In our preliminary experiments conducted in 2016, transgrafting on five 5-year-old transgenic 'Aurora' plants of three transgenic events promoted flower bud formation in non-transgenic scions. In this study, all transgenic VcFT-OX-Aurora plants were derived from one representative transgenic event which was reported in our previous studies ${ }^{45,47}$. Non-transgenic 'Aurora' and transgenic VcFT-OX-Aurora plants were obtained from in vitro cultured shoots. The shoots were transplanted to growing media for rooting in July 2015 and the rooted shoots were grown through the winter in a heated greenhouse from December 2015 to September 2016. The 2-year-old plants received natural chilling treatment in a courtyard between greenhouses from September 2016 to May 2017. The 3-year-old plants were used for transgrafting experiments in May 2017 through cleft grafting. Transgrafting and self-grafting were attempted three times, to produce a total of 12 pairs of grafts involving VcFT-OX-Aurora (noted as T thereafter) and non-transgenic 'Aurora' (noted as NT thereafter) for each of the following combinations: self-grafted transgenic VcFT-OX-Aurora T:T, self-grafted non-transgenic 'Aurosa' NT:NT, and transgrafted NT scion on T rootstock NT:T (Fig. 1; Fig. S4). In this study, T:NT did not survive and therefore no data were collected from the grafting. For each pair of $\mathrm{T}$ and NT plants, the selected shoot tips of two branches (one for $\mathrm{T}$ and the other for NT) were reciprocally grafted using splice grafting. Selfgrafting for $\mathrm{T}$ or NT plants was performed by cutting a 
shoot tip and rejoining the parts together. In each of these grafts, the leaves on the scions were removed and 5-10 leaves below the graft unions on the rootstocks were maintained. All grafted plants were grown for 3 weeks in a plant culture room at $25^{\circ} \mathrm{C}$ under 16 -h photoperiod of $35 \mu \mathrm{mol} \mathrm{m}{ }^{-2} \mathrm{~s}^{-1}$ light from cool white florescent tubes. The surviving scions were photo-identified before the plants were moved to the greenhouse. In September of 2017, six plants each of NT:T, NT:NT, T and NT were moved to a growth chamber for chilling treatment for 2 months at $4{ }^{\circ} \mathrm{C}$ under 10 -h photoperiod of $30 \mu \mathrm{mol} \mathrm{m}^{-2}$ $\mathrm{s}^{-1}$ light from cool white florescent tubes. Two each of NT:T and NT:NT grafted plants were grown in a greenhouse along with T and NT. Ten 6-year-old 'Aurora' plants grown in the courtyard were used as controls for monitoring 'Aurora' plant flowering. After receiving $\sim 1440 \mathrm{CU}$ in 2 months, the plants were moved to a greenhouse and grown at $22{ }^{\circ} \mathrm{C}$ under natural light conditions. Plant flowering was documented for all grafted plants as well as the controls from November 27, 2017 to May 23, 2018.

\section{Material for phytohormone and RNA-seq profiling}

Three chilled NT:T and NT:NT plants, representing three biological replicates, were used for phytohormone analysis and RNA-seq sequencing. Young roots and leaves from each plant were harvested in January 2018 in NT:T and NT:NT plants. Young leaves, 1-2 g per plant, were harvested from multiple new shoots; half of these leaves were subjected to freeze-drying immediately and the other half were ground in liquid nitrogen and stored in a $-80^{\circ} \mathrm{C}$ freezer. From each NT:T plant, transgenic leaves (on the rootstocks) and non-transgenic leaves (on the scions) were harvested, respectively. From each NT:NT plant, leaves from scions were harvested. Young roots, 1-2 g, were excised from each plant, washed in double distilled water and blotted dry on filter paper. Similarly, for each root sample, half were subjected to freeze-drying, and the other half were ground in liquid nitrogen and stored at $-80^{\circ} \mathrm{C}$.

\section{Phytohormone profiling}

Freeze-dried tissues were used for profiling $A B A$ and six ABA metabolites (ABAGE: Abscisic acid glucose ester, DPA: Dihydrophaseic acid, PA: Phaseic acid, 7'OH-ABA: 7'Hydroxy-abscisic acid, neo-PA: neo-Phaseic acid, and $t$ ABA: trans-Abscisic acid), auxins [IAA: Indole-3-acetic acid, IAA-Asp: N-(Indole-3-yl-acetyl)-aspartic acid, IAA-Glu: N(Indole-3-yl-acetyl)-glutamic acid, IAA-Ala: N-(Indole-3-ylacetyl)-alanine, IAA-Leu: $\mathrm{N}$-(Indole-3-yl-acetyl)-leucine, and IBA: Indole-3-butyric acid], cytokinins [t-ZOG: (trans) Zeatin-O-glucoside, $c$-ZOG: (cis) Zeatin-O-glucoside, $t$-Z: (trans) Zeatin, $c$-Z: (cis) Zeatin; dhZ: Dihydrozeatin, $t$-ZR: (trans) Zeatin riboside, $c$-ZR: (cis) Zeatin riboside, dhZR:
Dihydrozeatin riboside, iP: Isopentenyladenine, and iPR: Isopentenyladenosine], and 14 gibberellins (GA1, GA3, GA4, GA7, GA8, GA9, GA19, GA20, GA24, GA29, GA34, GA44, GA51, and GA53). These hormones were measured by the National Research Council of Canada, Saskatoon, SK S7N 0W9 (http://www.nrc-cnrc.gc.ca/eng/solutions/ advisory/plant_hormone.html).

Fresh tissues of the same set of materials were used for RNA-seq analysis and quantification of the hormones SA, JA, and two JA metabolites (MeJA: Methyl jasmonate, JAIle: jasmonoyl isoleucine). The samples for hormone quantification were prepared following the protocols for Arabidopsis $^{89}$ and were analyzed by the Mass Spectrometry and Metabolomics Core of Michigan State University. ANOVA and Tukey's test were conducted using RStudio (Version 1.0.136).

\section{RNA-seq and differential expression analysis}

Total RNA of each sample was isolated from $\sim 200 \mathrm{mg}$ of ground tissues using a CTAB method ${ }^{90}$, followed by using RNeasy Mini Kit for on-column DNase digestion and RNA purification (Qiagen, Valencia, CA, USA). The integrity of the RNA samples was assessed using the Agilent RNA 6000 Pico Kit (Agilent Technologies, Inc., Germany). All RNA samples submitted for RNA sequencing had an RNA quality score greater than 7.5 for roots and 8.0 for leaves. Sequencing (150-bp pair end reads) was conducted using the Illumina HiSeq4000 platform at the Research Technology Support Facility at Michigan State University (East Lansing, MI, USA). Thirty to sixty million reads were generated for each biological replicate. The FastQC program (www.bioinformatics. babraham.ac.uk/projects/fastqc/) was used to assess the quality of sequencing reads with the per base quality scores ranging from 30 to 40 .

The RNA-seq reads of three biological replicates for each type of tissues were analyzed using Trinity ${ }^{91}$. The paired reads were aligned to the transcriptome reference Reftrinity ${ }^{47}$, and the abundance for each of a single read was estimated using the Trinity command "align_and_estimate_abundance.pl". The Trinity command "run_DE_analysis.pl -method edgeR" was used to conduct differential expression analysis ${ }^{91}$. The differentially expressed (DE) genes or isoforms with the false discovery rate $(\mathrm{FDR})$ value below 0.05 ( $p$-value $<0.001)$ were used for further analyses of different pathway genes of blueberry. Fragments Per Kilobase of transcript per Million mapped reads (FPKM) were used to evaluate expression abundance. Most of the analyses were performed using the resources at the High Performance Computing Center at Michigan State University.

Pathway genes of nine phytohormones in Arabidopsis, including auxin, cytokinin, ABA, ethylene, gibberellin, brassinosteroid, jasmonic acid, salicylic acid, and 
strigolactones, were retrieved from RIKEN Plant Hormone Research Network and listed in Table S5. Similarly, pathway genes of sugar in Arabidopsis were identified and listed in Table S5. These Arabidopsis hormone and sugar genes were used as queries to blast against the transcriptome reference Reftrinity ${ }^{47}$ and the blueberry isoforms showing $E$-values less than -20 were identified and used for further analyses in various transcriptome comparisons. Blueberry flowering pathway genes identified in our previous study ${ }^{47}$ were used to analyze flowering-related DE isoforms identified in this study. Cytoscape 3.7.0 was used to construct gene networks of overrepresented gene ontology (GO) terms for the selected DETs under BiNGO's default parameters with selected ontology file 'GOSlim_Plants' and selected organism 'A. thaliana ${ }^{92,93}$.

\section{Acknowledgements}

We thank Dr. Xiaojuan Zong for blueberry grafting and collection of the plant tissues for hormone analysis.

\section{Author details \\ ${ }^{1}$ Plant Biotechnology Resource and Outreach Center, Department of Horticulture, Michigan State University, East Lansing, MI 48824, USA. ${ }^{2}$ Aquatic and Crop Resource Development, National Research Council of Canada, Saskatoon SK S7N OW9, Canada. ${ }^{3}$ Grape Genetics Research Unit, USDA-ARS, Geneva, NY 14456, USA}

\section{Conflict of interest}

The authors declare that they have no conflict of interest.

Supplementary Information accompanies this paper at (https://doi.org/ 10.1038/s41438-019-0188-5).

Received: 18 April 2019 Revised: 26 July 2019 Accepted: 29 July 2019 Published online: 11 September 2019

\section{References}

1. Bernier, G., Havelange, A., Houssa, C., Petitjean, A. \& Lejeune, P. Physiological signals that induce flowering. Plant Cell 5, 1147-1155 (1993).

2. Cajlachjan, M. C. \& Yarkovaja, L. M. New facts in support of the hormonal theory of plant development II. Comptes Rendus De L Academie Des Sciences De L URSS 15, 215-217 (1937).

3. Bernier, G. My favourite flowering image: the role of cytokinin as a flowering signal. J. Exp. Bot. 64, 5795-5799 (2013).

4. Kardailsky, I. et al. Activation tagging of the floral inducer FT. Science $\mathbf{2 8 6}$, 1962-1965 (1999).

5. Kobayashi, Y., Kaya, H., Goto, K, Iwabuchi, M. \& Araki, T. A pair of related genes with antagonistic roles in mediating flowering signals. Science 286, 1960-1962 (1999).

6. Pin, P. A. et al. An antagonistic pair of $\mathrm{FT}$ homologs mediates the control of flowering time in sugar beet. Science 330, 1397-1400 (2010).

7. Wickland, D. P. \& Hanzawa, Y. The FLOWERING LOCUS T/TERMINAL FLOWER 1 gene family: functional evolution and molecular mechanisms. Mol. Plant $\mathbf{8}$, 983-997 (2015)

8. Liu, Y. Y., Yang, K. Z., Wei, X. X. \& Wang, X. Q. Revisiting the phosphatidylethanolamine-binding protein (PEBP) gene family reveals cryptic FLOWERING LOCUS T gene homologs in gymnosperms and sheds new light on functional evolution. New Phytol. 212, 730-744 (2016).

9. Putterill, J. \& Varkonyi-Gasic, E. FT and florigen long-distance flowering control in plants. Curr. Opin. Plant Biol. 33, 77-82 (2016).

10. Liu, L., Zhu, Y., Shen, L. \& Yu, H. Emerging insights into florigen transport. Curr. Opin. Plant Biol. 16, 7 (2013).
11. Giakountis, A. \& Coupland, G. Phloem transport of flowering signals. Curr. Opin. Plant Biol. 11, 8 (2008).

12. Corbesier, L. et al. FT protein movement contributes to long-distance signaling in floral induction of Arabidopsis. Science 316, 1030-1033 (2007).

13. Notaguchi, M. et al. Long-distance, graft-transmissible action of Arabidopsis FLOWERING LOCUS T protein to promote flowering. Plant Cell Physiol. 49, 1645-1658 (2008)

14. Zhu, Y., Liu, L., Shen, L. \& Yu, H. NaKR1 regulates long-distance movement of Flowering Locus T in Arabidopsis. Nat. Plants 2, 16075 (2016).

15. Yoo, S. J., Hong, S. M., Jung, H. S. \& Ahn, J. H. The cotyledons produce sufficient $\mathrm{FT}$ protein to induce flowering: evidence from cotyledon micrografting in Arabidopsis. Plant Cell Physiol. 54, 119-128 (2013).

16. Jaeger, K. E. \& Wigge, P. A. FT protein acts as a long-range signal in Arabidopsis. Curr. Biol. 17, 5 (2007).

17. Mathieu, J., Warthmann, N., Küttner, F. \& Schmid, M. Export of FT protein from phloem companion cells is sufficient for floral induction in Arabidopsis. Curr. Biol. 17, 6 (2007).

18. Turck, F., Fornara, F. \& Coupland, G. Regulation and identity of florigen: FLOWERING LOCUS T moves center stage. Annu. Rev. Plant Biol. 59, 573-594 (2008).

19. Tamaki, S. et al. FT-like proteins induce transposon silencing in the shoot apex during floral induction in rice. Proc. Natl Acad. Sci. USA 112, E901-E910 (2015).

20. Lu, K. J., Huang, N. C., Liu, Y. S., Lu, C. A. \& Yu, T. S. Long-distance movement of Arabidopsis FLOWERING LOCUS T RNA participates in systemic floral regulation. RNA Biol. 9, 653-662 (2012).

21. Huang, N. C., Luo, K. R. \& Yu, T. S. Mobility of antiflorigen and PEBP mRNAs in tomato-tobacco heterografts. Plant Physiol. 178, 783-794 (2018).

22. $\mathrm{Ye}$, J. et al. The Jatropha FT ortholog is a systemic signal regulating growth and flowering time. Biotechnol. Biofuels 71, 10 (2014).

23. Bull, S. E. et al. FLOWERING LOCUS T triggers early and fertile flowering in glasshouse cassava (Manihot esculenta Crantz). Plants (Basel) 6, 10.3390/ plants6020022 (2017).

24. Zhang, H. et al. Precocious flowering in trees: the FLOWERING LOCUS T gene as a research and breeding tool in Populus. J. Exp. Bot. 61, 2549-2560 (2010).

25. Wenzel, S., Flachowsky, H. \& Hanke, M.-V. The Fast-track breeding approach can be improved by heat-induced expression of the FLOWERING LOCUS T genes from poplar (Populus trichocarpa) in apple (Malus $\times$ domestica Borkh.). Plant Cell, Tissue Organ Cult. (PCTOC) 115, 127-137 (2013).

26. Srinivasan, C., Dardick, C., Callahan, A. \& Scorza, R. Plum (Prunus domestica) trees transformed with poplar FT1 result in altered architecture, dormancy requirement, and continuous flowering. PLOS ONE 7, e40715 (2012).

27. Ionescu, I. A., Moller, B. L. \& Sanchez-Perez, R. Chemical control of flowering time. J. Exp. Bot. 68, 369-382 (2017).

28. Fornara, F., de Montaigu, A. \& Coupland, G. SnapShot: control of flowering in Arabidopsis. Cell 141, https://doi.org/10.1016/j.cell.2010.04.024 (2010).

29. Busov, $\mathrm{V}$. et al. Transgenic modification of gai or rgl1 causes dwarfing and alters gibberellins, root growth, and metabolite profiles in Populus. Planta 224, 288-299 (2006).

30. Hartweck, L. M. Gibberellin signaling. Planta 229, 1-13 (2008).

31. Daviere, J. M. \& Achard, P. Gibberellin signaling in plants. Development $\mathbf{1 4 0}$ 1147-1151 (2013).

32. Ji, S. H., Gururani, M. A., Lee, J. W., Ahn, B. O. \& Chun, S. C. Isolation and characterisation of a dwarf rice mutant exhibiting defective gibberellins biosynthesis. Plant Biol. (Stuttg.) 16, 428-439 (2014).

33. Yamaguchi, N. et al. A molecular framework for auxin-mediated initiation of flower primordia. Dev. Cell 24, 271-282 (2013).

34. Krizek, B. A. Auxin regulation of Arabidopsis flower development involves members of the AINTEGUMENTA-LIKE/PLETHORA (AIL/PLT) family. J. Exp. Bot. 62, 3311-3319 (2011)

35. Osugi, A. \& Sakakibara, H. Q\&A: How do plants respond to cytokinins and what is their importance? BMC Biol. 13, 102 (2015).

36. Ren, B. et al. Genome-wide comparative analysis of type-A Arabidopsis response regulator genes by overexpression studies reveals their diverse roles and regulatory mechanisms in cytokinin signaling. Cell Res. 19, 1178-1190 (2009).

37. Achard, P. et al. The plant stress hormone ethylene controls floral transition via DELLA-dependent regulation of floral meristem-identity genes. Proc. Natl Acad. Sci. USA 104, 6484-6489 (2007).

38. Domagalska, M. A. et al. Attenuation of brassinosteroid signaling enhances FLC expression and delays flowering. Development 134, 2841-2850 (2007). 
39. Kutschera, U. \& Wang, Z. Y. Brassinosteroid action in flowering plants: a Darwinian perspective. J. Exp. Bot. 63, 3511-3522 (2012)

40. Ishiguro, S., Kawai-Oda, A., Ueda, J., Nishida, I. \& Okada, K. The DEFECTIVE IN ANTHER DEHISCIENCE gene encodes a novel phospholipase A1 catalyzing the initial step of jasmonic acid biosynthesis, which synchronizes pollen maturation, anther dehiscence, and flower opening in Arabidopsis. Plant Cell $\mathbf{1 3}$ 2191-2209 (2001).

41. Takahash, M. \& Morikawa, H. Nitrogen dioxide accelerates flowering without changing the number of leaves at flowering in Arabidopsis thaliana. Plant Signaling \& Behavior 9, 10.4161/15592316.2014.970433 (2014).

42. Wang, Y. H. \& Irving, H. R. Developing a model of plant hormone interactions. Plant Signal Behav. 6, 494-500 (2011).

43. Martinez, C., Pons, E., Prats, G. \& Leon, J. Salicylic acid regulates flowering time and links defence responses and reproductive development. Plant J. 37, 209-217 (2004).

44. Rivas-San Vicente, M. \& Plasencia, J. Salicylic acid beyond defence: its role in plant growth and development. J. Exp. Bot. 62, 3321-3338 (2011).

45. Gao, X., Walworth, A. E., Mackie, C. \& Song, G. Q. Overexpression of blueberry FLOWERING LOCUS T is associated with changes in the expression of phytohormone-related genes in blueberry plants. Hortic. Res. 3, 16053 (2016).

46. Song, G. Q., Walworth, A., Zhao, D. Y., Jiang, N. \& Hancock, J. F. The Vaccinium corymbosum FLOWERING LOCUS T-like gene (VCFT): a flowering activator reverses photoperiodic and chilling requirements in blueberry. Plant Cell Rep. 32, 1759-1769 (2013).

47. Walworth, A. E., Chai, B. \& Song, G. Q. Transcript profile of flowering regulatory genes in VCFT-overexpressing blueberry plants. PLOS ONE 11, e0156993 (2016).

48. Casamali, B., Darnell, R. L., Kovaleski, A. P., Olmstead, J. W. \& Williamson, J. G. Vegetative and reproductive traits of two southern highbush blueberry cultivars grafted onto Vaccinium arboreum rootstocks. Hortscience 51, 880-886 (2016).

49. King, R. W. et al. Regulation of flowering in the long-day grass Lolium temulentum by gibberellins and the FLOWERING LOCUS T gene. Plant Physiol. 141, 498-507 (2006)

50. Remay, A. et al. A survey of flowering genes reveals the role of gibberellins in floral control in rose. Theor. Appl Genet 119, 767-781 (2009).

51. Bouche, F. et al. Integrating roots into a whole plant network of flowering time genes in Arabidopsis thaliana. Sci Rep-Uk 6, 29042 (2016).

52. Lee, J. \& Lee, I. Regulation and function of SOC1, a flowering pathway integrator. J. Exp. Bot. 61, 2247-2254 (2010).

53. Xing, D., Zhao, H., Xu, R. \& Li, Q. Q. Arabidopsis PCFS4, a homologue of yeast polyadenylation factor Pcf11p, regulates FCA alternative processing and promotes flowering time. Plant J. 54, 899-910 (2008).

54. Kiba, T., Takei, K., Kojima, M. \& Sakakibara, H. Side-chain modification of cytokinins controls shoot growth in Arabidopsis. Dev. Cell 27, 452-461 (2013).

55. Jin, S. H. et al. Overexpression of glucosyltransferase UGT85A1 influences transzeatin homeostasis and trans-zeatin responses likely through O-glucosylation. Planta 237, 991-999 (2013).

56. Sudre, D. et al. Iron-dependent modifications of the flower transcriptome, proteome, metabolome, and hormonal content in an Arabidopsis ferritin mutant. J. Exp. Bot. 64, 2665-2688 (2013).

57. Ionescu, I. A. et al. Transcriptome and metabolite changes during hydrogen cyanamide-induced floral bud break in sweet cherry. Front. Plant Sci. 8, 1233 (2017).

58. Schafer, M. et al. The role of cis-zeatin-type cytokinins in plant growth regulation and mediating responses to environmental interactions. J. Exp. Bot. 66, 4873-4884 (2015).

59. Thomas, S. G., Phillips, A. L. \& Hedden, P. Molecular cloning and functional expression of gibberellin 2-oxidases, multifunctional enzymes involved in gibberellin deactivation. Proc. Natl Acad. Sci. USA 96 4698-4703 (1999).

60. Schomburg, F. M., Bizzell, C. M., Lee, D. J., Zeevaart, J. A. \& Amasino, R. M. Overexpression of a novel class of gibberellin 2-oxidases decreases gibberellin levels and creates dwarf plants. Plant Cell 15, 151-163 (2003).
61. Zhao, X. Y. et al. Over-expression of the AtGA2ox8 gene decreases the biomass accumulation and lignification in rapeseed (Brassica napus L.). J. Zhejiang Univ. Sci. B 11, 471-481 (2010).

62. Rieu, I. et al. The gibberellin biosynthetic genes AtGA20ox1 and AtGA20ox2 act, partially redundantly, to promote growth and development throughout the Arabidopsis life cycle. Plant J. 53, 488-504 (2008).

63. King, R. W. et al. Selective deactivation of gibberellins below the shoot apex is critical to flowering but not to stem elongation of lolium. Mol. Plant 1, 9 (2008).

64. Dong, T., Park, Y. \& Hwang, I. Abscisic acid: biosynthesis, inactivation, homoeostasis and signalling. Essays Biochem. 58, 29-48 (2015).

65. Nafisi, M. et al. Arabidopsis cytochrome P450 monooxygenase 71A13 catalyzes the conversion of indole-3-acetaldoxime in camalexin synthesis. Plant Cell 19 2039-2052 (2007)

66. Stepanova, A. N. et al. The Arabidopsis YUCCA1 flavin monooxygenase functions in the indole-3-pyruvic acid branch of auxin biosynthesis. Plant Cell 23, 3961-3973 (2011).

67. Mashiguchi, K. et al. The main auxin biosynthesis pathway in Arabidopsis. Proc. Natl Acad. Sci. USA 108, 18512-18517 (2011).

68. Nakamura, M. et al. Activation of the cytochrome P450 gene, CYP72C1, reduces the levels of active brassinosteroids in vivo. J. Exp. Bot. 56, 833-840 (2005).

69. Bai, S. L. et al. Transcriptome analysis of japanese pear (Pyrus pyrifolia Nakai) flower buds transitioning through endodormancy. Plant Cell Physiol. 54, 1132-1151 (2013)

70. Li, J., Li, Y., Chen, S. \& An, L. Involvement of brassinosteroid signals in the floralinduction network of Arabidopsis. J. Exp. Bot. 61, 4221-4230 (2010).

71. George Thompson, A. M., lancu, C. V., Neet, K. E., Dean, J. V. \& Choe, J. Y. Differences in salicylic acid glucose conjugations by UGT74F1 and UGT74F2 from Arabidopsis thaliana. Sci. Rep. 7, 46629 (2017).

72. Vlot, A. C. et al. Identification of likely orthologs of tobacco salicylic acidbinding protein 2 and their role in systemic acquired resistance in Arabidopsis thaliana. Plant J. 56, 445-456 (2008).

73. Widemann, E., Smirnova, E., Aubert, Y., Miesch, L. \& Heitz, T. Dynamics of jasmonate metabolism upon flowering and across leaf stress responses in Arabidopsis thaliana. Plants (Basel) 5, 15 (2016).

74. Kandel, S. et al. Characterization of a methyl jasmonate and woundingresponsive cytochrome P450 of Arabidopsis thaliana catalyzing dicarboxylic fatty acid formation in vitro. FEBS J. 274, 5116-5127 (2007).

75. Chehab, E. W. et al. Intronic T-DNA insertion renders Arabidopsis opr3 a conditional jasmonic acid-producing mutant. Plant Physiol. 156, 770-778 (2011).

76. Koo, A. J., Chung, H. S., Kobayashi, Y. \& Howe, G. A. Identification of a peroxisomal acyl-activating enzyme involved in the biosynthesis of jasmonic acid in Arabidopsis. J. Biol. Chem. 281, 33511-33520 (2006).

77. Walworth, A., Wickenheiser, R. \& Song, G. Rootstock-specific expression of VcFT in tobacco is insufficient to alter scion flowering regime. In vitro Cell Dev. Plant, 521 (2014).

78. Chen, Q. G. et al. FLOWERING LOCUS T mRNA is synthesized in specialized companion cells in Arabidopsis and Maryland Mammoth tobacco leaf veins. Proc. Natl Acad. Sci. USA 115, 2830-2835 (2018).

79. Farrona, S. et al. Tissue-specific expression of FLOWERING LOCUS T in Arabidopsis is maintained independently of polycomb group protein repression. Plant Cell 23, 3204-3214 (2011).

80. Song, G. Q., Walworth, A. E. \& Loescher, W. H. Grafting of genetically engineered plants. J. Am. Soc. Hortic. Sci. 140, 203-213 (2015).

81. Qin, Z., Bai, Y. \& Wu, L. Flowering on time: multilayered restrictions on $\mathrm{FT}$ in plants. Mol. Plant 10, 1365-1367 (2017).

82. Koshita, Y., Takahara, T., Ogata, T. \& Goto, A. Involvement of endogenous plant hormones (IAA, ABA, GAs) in leaves and flower bud formation of satsuma mandarin (Citrus unshiu Marc.). Sci. Hortic.-Amst. 79, 185-194 (1999).

83. Ohto, M. et al. Effects of sugar on vegetative development and floral transition in Arabidopsis. Plant Physiol. 127, 252-261 (2001).

84. Barbier, F. et al. Sucrose is an early modulator of the key hormonal mechanisms controlling bud outgrowth in Rosa hybrida. J. Exp. Bot. 66, 2569-2582 (2015) 
85. Arrom, L. \& Munne-Bosch, S. Hormonal changes during flower development in floral tissues of Lilium. Planta 236, 343-354 (2012).

86. Arrom, L. \& Munne-Bosch, S. Sucrose accelerates flower opening and delays senescence through a hormonal effect in cut lily flowers. Plant Sci. 188, 41-47 (2012).

87. Bolouri Moghaddam, M. R. \& Van den Ende, W. Sugars, the clock and transition to flowering. Front Plant Sci. 4, 22 (2013).

88. Wilkie, J. D., Sedgley, M. \& Olesen, T. Regulation of floral initiation in horticultural trees. J. Exp. Bot. 59, 3215-3228 (2008).

89. Zeng, W. Q. et al. A genetic screen reveals Arabidopsis stomatal and/or apoplastic defenses against Pseudomonas syringae pv. tomato DC3000. Plos. Pathog. 7, e1002291 (2011)
90. Zamboni, A., Pierantoni, L. \& De Franceschi, P. Total RNA extraction from strawberry tree (Arbutus unedo) and several other woodyplants. IforestBiogeosciences For. 1, 122-125 (2008).

91. Haas, B. J. et al. De novo transcript sequence reconstruction from RNA-seq using the Trinity platform for reference generation and analysis. Nat. Protoc. $\mathbf{8}$, 1494-1512 (2013)

92. Shannon, P. et al. Cytoscape: a software environment for integrated models of biomolecular interaction networks. Genome Res. 13, 2498-2504 (2003).

93. Maere, S., Heymans, K. \& Kuiper, M. BiNGO: a Cytoscape plugin to assess overrepresentation of gene ontology categories in biological networks. Bioinformatics 21, 3448-3449 (2005). 